\title{
Some Results on Disturbance Attenuation for Hamiltonian Systems via Direct Discrete-Time Design
}

\author{
Yaprak Yalçın ${ }^{1}$, Leyla Gören-Sümer ${ }^{1}$, Alessandro Astolfi ${ }^{2}$ \\ ${ }^{l}$ Control Engineering Department, Istanbul Technical University, Istanbul, Turkey. \\ ${ }^{2}$ Control and Power Research Group, Department of Electric and Electronics, Imperial College, London, UK.
}

SUMMARY

The disturbance attenuation and robust disturbance attenuation problems for Hamiltonian systems in the discrete-time setting are considered and some new results are presented. The new results are derived utilizing the recently presented dissipativity equality obtained by adding the dissipation rate function to the classical dissipativity inequality. A selection of the dissipation rate function yields the new results. These results include a condition on the dissipation structure of the system to achieve the desired disturbance attenuation level and gives direct construction of optimal control laws for any desired disturbance attenuation level. The results remove the need to solve Hamilton-Jacobi-Isaacs inequalities.

KEY WORDS: Hamiltonian Systems, Nonlinear Systems, Disturbance Attenuation, $\mathrm{H}_{\infty}$ Optimal Control, Robust Control.

\section{INTRODUCTION}

The subject of the modelling and control of complex nonlinear systems has been an important and active area of research in control theory and its applications. In this context, the port-controlled Hamiltonian (PCH) approach has been introduced for the modelling and control of nonlinear systems, especially when electrical and mechanical sub-systems are considered. There is large number of publications on this subject, see for example [2, 3, 4].

In the literature, the problem of local disturbance attenuation for general continuous time nonlinear systems has been studied comprehensively with the $H_{\infty}$ approach, see [5, 6, 7]. In [8, 9] some nonlinear $H_{\infty}$ control problems for continuous time Hamiltonian systems have been considered and some results have been presented. The results therein demonstrate that while the disturbance attenuation problem characterized by means of the $L_{2}$ gain requires solving the Hamilton-JacobiIsaacs (HJI) partial differential inequality for general continuous-time nonlinear systems, it can be reduced to solve an algebraic Hamilton-Jacobi-Isaacs (HJI) inequality for Hamiltonian systems.

The development of modelling and control techniques for discrete time nonlinear systems has been increasingly important since in engineering practice computer-controlled systems are often preferred. There are various studies on discrete time nonlinear systems in the literature. These studies can be classified, roughly, in two groups. One group deals with the concepts of losslessness, feedback equivalence and the global stabilization of discrete-time nonlinear systems $[1,10,11,12,13]$; the second deals with the derivation of discrete-time counterpart of the $H_{\infty}$ control technique which is developed using the model of the system [11].

Additionally, results on the stabilization problem for general sampled-data nonlinear systems using their approximate discrete-time models have been given, for example, in [14] and a direct discretetime PBC (Passivity Based Control) method for approximate discrete-time Hamiltonian models have 
been developed in $[15,16,17]$. A discrete-time counterpart of the PBC technique has been developed for n-degrees of freedom mechanical systems in $[18,19]$. In this study, some new results on the disturbance attenuation and robust disturbance attenuation problems for Hamiltonian systems are presented in the discrete-time setting. In our previous publications [20, 21] the discrete time disturbance attenuation problem and robust disturbance attenuation problem for n-dof Hamiltonian systems with energy function uncertainty have been considered. This problem has been formulated for the discrete-time Hamiltonian model of the system and sufficient conditions for the existence of the solutions of the problems have been given. It has been shown that the solution of the problem can be reduced to an algebraic $\mathrm{HJI}$ inequality.

In this study the disturbance attenuation and robust disturbance attenuation problems for Hamiltonian systems are reconsidered using the concepts of dissipation rate and dissipativity equality presented by Navarro-Lopez in [1] and some new results - that do not require solving an inequality as in our previous studies- are presented. The result presented reveals the relation between the dissipation structure and the disturbance attenuation level of the system, that is to say a condition is presented that gives a lower bound on the dissipation structure of the system to have any specific level of disturbance attenuation. The optimal controller construction assigning a dissipation structure that satisfies this condition is given. Moreover a relation between this control laws and dissipation injection control laws is also established.

In the sequel, the notation $x_{k}=x(k), w_{k}=w(k), y_{k}=y(k), u_{k}=u(k), q_{k}=q(k), p_{k}=p(k)$ is used.

\section{PRELIMINARIES}

This section is devoted to the definitions of dissipativity concepts for nonlinear systems $[1,11]$ and the bounded real lemma [11] used to solve the $H_{\infty}$ control problem for input affine discrete-time nonlinear systems described by equations of the form

$$
\begin{aligned}
& x_{k+1}=f\left(x_{k}\right)+g_{1}\left(x_{k}\right) w_{k}+g_{2}\left(x_{k}\right) u_{k}, \\
& z_{k}=h_{1}\left(x_{k}\right)+k_{11}\left(x_{k}\right) w_{k}+k_{21}\left(x_{k}\right) u_{k}, \\
& y_{k}=h_{2}\left(x_{k}\right)+k_{12}\left(x_{k}\right) w_{k},
\end{aligned}
$$

where $x_{k} \in R^{n}$ is the state vector, $y_{k} \in R^{r}$ is the system output, $z_{k}$ is the penalty signal and $w_{k} \in R^{m_{1}}$, $u_{k} \in R^{m_{2}}$ are the disturbance and control inputs, respectively. The use of static feedback control law $u=c\left(x_{k}\right)$ yields a closed-loop system of the form,

$$
\begin{aligned}
& x_{k+1}=f_{c l}\left(x_{k}, w_{k}\right), \\
& z_{k}=h_{c l}\left(x_{k}, w_{k}\right) .
\end{aligned}
$$

Consider now the following definition.

\section{Definition 1 [11]}

The discrete-time nonlinear system (2) is said to be dissipative with supply rate $s\left(z_{k}, w_{k}\right)=1 / 2\left(\gamma^{2}\left\|w_{k}\right\|^{2}-\left\|z_{k}\right\|^{2}\right)$ if there exists a nonnegative function $V: X \rightarrow R^{+}, X \in R^{n}$ with $V(0)=0$, which is called storage function, such that $\forall w_{k} \in R^{m_{1}}$ and $k=0,1,2, \ldots$. the inequality

$$
V\left(x_{k+1}\right)-V\left(x_{k}\right) \leq \frac{1}{2}\left(\gamma^{2}\left\|w_{k}\right\|^{2}-\left\|z_{k}\right\|^{2}\right)
$$


is satisfied.

\section{Definition 2 [11]}

Let $\gamma>0$. The nonlinear system (2) is said to have $L_{2}$ gain less than or equal to $\gamma$, from the input $w_{k}$ to the output $z_{k}$ if,

$$
\sum_{k=0}^{N}\left\|z_{k}\right\|^{2} \leq \gamma^{2} \sum_{k=0}^{N}\left\|w_{k}\right\|^{2}
$$

$\forall N \in Z_{+}:=1,2,3, \ldots \ldots .$. and $\forall w_{k} \in l_{2}\left(0, N, R^{m_{1}}\right)$

\section{Lemma (Bounded Real Lemma) [11]}

Suppose that the discrete-time nonlinear system (2) with $w_{k}=0$ has $x=0$ as a locally asymptotically stable equilibrium. Then the system has an $L_{2}$ gain less than or equal to $\gamma$ if it is dissipative with a positive definite storage function $V$ with respect to the supply rate $1 / 2\left(\gamma^{2}\left\|w_{k}\right\|^{2}-\left\|z_{k}\right\|^{2}\right)$. Conversely, suppose that system (2) has an $L_{2}$ gain less than or equal to $\gamma$ and (2) is reachable from $x=0$. Then the system (2) is dissipative with a positive definite storage function $V$ with respect to the supply rate $1 / 2\left(\gamma^{2}\left\|w_{k}\right\|^{2}-\left\|z_{k}\right\|^{2}\right)$.

In what follows the definitions presented in [1] are restated for the nonlinear system (2).

\section{Definition 3 [1]}

The system (2) is said to be dissipative (respectively, strictly dissipative) with respect to a real valued function $s\left(z_{k}, w_{k}\right)$ called the supply (rate) function if there exist a positive definite function, $V: X \rightarrow R^{+}$, with $V(0)=0$, called the storage function, and a continuous function $\varphi: X \times W \rightarrow \mathfrak{R}^{+}$where $W \in R^{q}$ with $\varphi(., w)$ is positive (respectively, strictly positive) for each $w \in W$ such that

$$
V\left(x_{k+1}\right)-V\left(x_{k}\right)=s\left(z_{k}, w_{k}\right)-\varphi\left(x_{k}, w_{k}\right)
$$

for all $w \in W$ and $k=0,1,2, \ldots$ irrespectively of the initial value of the state. The function $\varphi(., w)$ is called the dissipation rate function.

\section{Definition 4}

A $C^{2}$ function $\varphi: X \times W \rightarrow \mathfrak{R}^{+}$, such that $\varphi(., w)$ is positive (respectively, strictly positive) for each $w \in W$ and $\varphi(0,0)=0$, is a dissipation rate (resp. strict dissipation rate) function in the sense in (Hill and Moylan, 1980).

\section{Remark 1}

Using the supply rate function $s=1 / 2\left(\gamma^{2}\left\|w_{k}\right\|^{2}-\left\|z_{k}\right\|^{2}\right)$ in (7) yields 


$$
V\left(x_{k+1}\right)-V\left(x_{k}\right)=\frac{1}{2}\left(\gamma^{2}\left\|w_{k}\right\|^{2}-\left\|z_{k}\right\|^{2}\right)-\varphi\left(x_{k}, w_{k}\right)
$$

It is easy to see the correspondence of this equality with the inequality (3), namely, when the equality (6) holds the inequality (3) is always satisfied, and vice-versa.

We finally recall the discrete gradient conditions [22], since a gradient-based discrete time model of the considered class of Hamiltonian systems is used.

\section{Definition 6 [22]}

Let $H(x)$ be a differentiable scalar function of $x . \bar{\nabla} H\left(x_{k+1}, x_{k}\right)$ is a discrete gradient of $H$ if it is continuous in $x$ and

$$
\begin{aligned}
\bar{\nabla} H\left(x_{k+1}, x_{k}\right)^{T} x_{k+1}-x_{k} & =H\left(x_{k+1}\right)-H\left(x_{k}\right), \\
\bar{\nabla} H\left(x_{k}, x_{k}\right) & =\nabla H\left(x_{k}\right) .
\end{aligned}
$$

\section{PROBLEM FORMULATION}

\subsection{Disturbance Attenuation}

The direct discrete-time disturbance attenuation problem formulation given in [20] is restated below. The considered class of Hamiltonian systems is given by the equations

$$
\begin{aligned}
{\left[\begin{array}{c}
\dot{q} \\
\dot{p}
\end{array}\right] } & =(J-R(q, p)) \nabla H(q, p)+G_{1}(q) w+G_{2}(q) u \\
y(t) & =G_{1}^{T}(q) \nabla H(q, p)
\end{aligned}
$$

where $x=(q, p) \in \mathfrak{R}^{2 n}$ is the state vector, $w \in \mathfrak{R}^{m_{1}}$ is the disturbance input, $u \in \mathfrak{R}^{m_{2}}$ is the control input, $y \in \mathfrak{R}^{m_{1}}$ is the output, $J$ is a skew-symmetric interconnection matrix and $R(q, p) \in \mathfrak{R}^{2 n \times 2 n}$ is nonnegative symmetric matrix describing the dissipation structure, given by,

$$
\begin{aligned}
& J=\left[\begin{array}{cc}
0 & I_{n} \\
-I_{n} & 0
\end{array}\right], R(q, p)=\left[\begin{array}{cc}
0 & 0 \\
0 & R_{1}(q, p)
\end{array}\right] \text { with } \\
& R_{1}(q, p) \in R^{n x n} \geq 0, \text { and } G_{1}(q)=\left[\begin{array}{c}
0 \\
g_{1}(q)
\end{array}\right], G_{2}(q)=\left[\begin{array}{c}
0 \\
g_{2}(q)
\end{array}\right]
\end{aligned}
$$

in which $g_{1}(q) \in \mathfrak{R}^{n x m_{1}}, g_{2}(q) \in \mathfrak{R}^{n x m_{2}}$ are the disturbance input matrix and the control input matrix, respectively. The notation $\nabla_{(\bullet)} H$ is used for the gradient vector of a scalar function $H(q, p)$ with respect to the arguments (.). $H: \mathfrak{R}^{2 n} \rightarrow \mathfrak{R}$ is the Hamiltonian energy function of the system and is given by,

$$
H(q, p)=\frac{1}{2} p^{T} M(q)^{-1} p+P(q)
$$


where $M(q)=M(q)^{T}>0$ is the generalized inertia matrix and $P(q)$ is the potential energy function of the system.

Employing the discrete-time modelling method given in $[18,19]$ the discrete-time description of the system (9) yields

$$
\begin{aligned}
{\left[\begin{array}{c}
q_{k+1}-q_{k} \\
p_{k+1}-p_{k}
\end{array}\right] } & =T\left(J-R\left(q_{k}, p_{k}\right)\right) \bar{\nabla} H+T G_{I}\left(q_{k}\right) w_{k}+T G_{2}\left(q_{k}\right) u_{k}, \\
y_{k} & =G_{l}^{T}\left(q_{k}\right) \bar{\nabla} H,
\end{aligned}
$$

where $T$ is the sampling period with $\bar{\nabla} H\left(x_{k+1}, x_{k}\right)$ is the discrete gradient of $H(x), x=(q, p)$, $x_{k}=\left(q_{k}, p_{k}\right)$ and $x_{k+1}=\left(q_{k+1}, p_{k+1}\right)$. The penalty signal is defined as

$$
z\left(q_{k}, p_{k}\right)=\left[\begin{array}{c}
y_{k} \\
d_{k}\left(q_{k}, p_{k}\right) u_{k}
\end{array}\right]
$$

and the disturbance rejection performance is described by the condition

$$
\sum_{k=0}^{N}\left\{y_{k}^{T} y_{k}+u_{k}^{T} D\left(q_{k}, p_{k}\right) u_{k}\right\} \leq \gamma^{2} \sum_{k=0}^{N}\left\|w_{k}\right\|^{2}
$$

where $D\left(q_{k}, p_{k}\right)=d^{T}\left(q_{k}, p_{k}\right) d\left(q_{k}, p_{k}\right)$ is a given weighting matrix, with $d\left(q_{k}, p_{k}\right)$ full rank, and $\gamma$ is the disturbance attenuation level.

The discrete time disturbance attenuation problem consists in finding a control law $u_{k}=c\left(q_{k}, p_{k}\right)$ such that the zero equilibrium of system with $w_{k}=0$ is asymptotically stable and the closed loop system satisfies the disturbance rejection performance given in (12), namely the $L_{2}$ gain from the disturbance input $w_{k}$ to the penalty signal $z_{k}$ is equal or less than the prescribed level $\gamma$.

\subsection{Robust Disturbance Attenuation}

In [21] it has been shown that the robust control problem for n-dof mechanical systems can be reduced to a disturbance attenuation problem. The port-controlled Hamiltonian formulation of the considered class of uncertain systems is

$$
\begin{aligned}
& {\left[\begin{array}{c}
\dot{q} \\
\dot{p}
\end{array}\right]=(J-R(q, p)) \nabla \tilde{H}+G_{w}(q) w_{e}(t)+G_{u}(q) u(t)} \\
& y(t)=G_{w}^{T}(q) \nabla \tilde{H}
\end{aligned}
$$

with

$$
G_{w}(q)=\left[\begin{array}{c}
0 \\
g_{w}(q)
\end{array}\right], G_{u}(q)=\left[\begin{array}{c}
0 \\
g_{u}(q)
\end{array}\right]
$$

where $\tilde{H}=H-\Delta H$,

$$
H(q, p)=\frac{1}{2} p^{T} M(q)^{-1} p+P(q)
$$




$$
\Delta H(q, p)=\frac{1}{2} p^{T} \Delta M(q)^{-1} p+\Delta P(q) .
$$

In [21] the robust disturbance attenuation problem has been formulated as a disturbance attenuation problem in which two disturbance inputs affect the system, i.e.

$$
\begin{aligned}
{\left[\begin{array}{c}
\dot{q} \\
\dot{p}
\end{array}\right] } & =(J-R(q, p)) \nabla H+G_{u}(q) w_{\Delta}(t)+G_{w}(q) w_{e}(t)+G_{u}(q) u(t), \\
y(t) & =G_{u}(q) \quad G_{w}(q)^{T} \nabla H,
\end{aligned}
$$

where $w_{\Delta}(t)$ and $w_{e}(t)$ are the disturbances corresponding to the uncertainty in the energy function and the external disturbance, respectively. The discrete time equations of the system (16) can be written in the form (10), i.e.

$$
\begin{aligned}
{\left[\begin{array}{c}
q_{k+1}-q_{k} \\
p_{k+1}-p_{k}
\end{array}\right] } & =T\left(J-R\left(q_{k}, p_{k}\right)\right) \bar{\nabla} H+T G_{1}\left(q_{k}\right) w_{k}+T G_{2}\left(q_{k}\right) u_{k}, \\
y_{k} & =G_{1}^{T}\left(q_{k}\right) \bar{\nabla} H,
\end{aligned}
$$

where

$$
\begin{aligned}
w_{k} & =w(k)=\left[\begin{array}{ll}
w_{\Delta}(k) & w_{e}(k)
\end{array}\right]^{T} \\
G_{1}\left(q_{k}\right) & =G_{u}\left(q_{k}\right) \quad G_{w}\left(q_{k}\right), G_{2}\left(q_{k}\right)=G_{u}\left(q_{k}\right) .
\end{aligned}
$$

Thus the discrete-time robust disturbance attenuation problem for the system (13) can be formulated as the disturbance attenuation problem for the system (17).

\subsection{Previous Results}

In this section the main results of our previous studies $[20,21]$ are summarized to exploit them in the sequel while obtaining the new results. In $[20,21]$, it has been shown that a static feedback of the form

$$
u_{k}=-\alpha T\left\{d_{k}^{T} d_{k}\right\}^{-1} g_{u}^{T}\left(q_{k}\right) \bar{\nabla}_{p} H
$$

solves the disturbance attenuation problem, if there exists a positive real number $\alpha$ satisfying the inequality

$$
\begin{aligned}
& \alpha^{2} \gamma^{-2} T^{2} g_{1}\left(q_{k}\right) g_{1}^{T}\left(q_{k}\right)-\alpha^{2} T^{2} g_{2}\left(q_{k}\right)\left\{d_{k}^{T} d_{k}\right\}^{-1} g_{2}^{T}\left(q_{k}\right)- \\
& -2 \alpha T R_{1}\left(q_{k}, p_{k}\right)+g_{1}\left(q_{k}\right) g_{1}^{T}\left(q_{k}\right) \leq 0
\end{aligned}
$$

for a prescribed $\gamma$. Moreover, the robust disturbance attenuation problem is solvable if the condition

$$
\begin{gathered}
\alpha^{2} \gamma^{-2} T^{2} g_{u}\left(q_{k}\right) g_{u}^{T}\left(q_{k}\right)+g_{w}\left(q_{k}\right) g_{w}^{T}\left(q_{k}\right)- \\
-\alpha^{2} T^{2} g_{u}\left(q_{k}\right)\left\{d_{k}^{T} d_{k}\right\}^{-1} g_{u}^{T}\left(q_{k}\right)- \\
-2 \alpha T R_{1}\left(q_{k}, p_{k}\right)+g_{u}\left(q_{k}\right) g_{u}^{T}\left(q_{k}\right)+g_{w}\left(q_{k}\right) g_{w}^{T}\left(q_{k}\right) \leq 0
\end{gathered}
$$

is satisfied for any $\alpha$. To construct the control law (19), a value of $\alpha$ satisfying (20) or (21) should be found. There is no direct method to obtain such an $\alpha$. 


\section{MAIN RESULTS}

This section presents the new results on the disturbance attenuation and robust disturbance attenuation problems. The following proposition gives a lower bound on the "dissipation" that the system (10)-(11) needs to have to be able to attenuate the effect of disturbance on the output to the level $\gamma>0$.

\section{Proposition 1}

Consider the discrete time port-controlled Hamiltonian system (10)-(11) with $u_{k}=0$, $k=0,1,2, \ldots$. If

$$
\underline{\sigma}\left(R_{1}\left(q_{k}, p_{k}\right)\right) \geq \bar{\sigma}\left(\frac{\left(1+\gamma^{-2}\right)}{2} g_{1}\left(q_{k}\right) g_{1}^{T}\left(q_{k}\right)\right), \forall q_{k}, p_{k}
$$

then the $L_{2}$ gain of the system, from the disturbance input $w_{k}$ to the penalty output $z_{k}$ defined in (11) is less than or equal to $\gamma$, namely the condition

$$
\sum_{k=0}^{N}\left\|z_{k}\right\|^{2} \leq \gamma^{2} \sum_{k=0}^{N}\left\|w_{k}\right\|^{2}
$$

holds. $\square$

Proof

The dissipation equality (5) for the system (10)-(11) with $s=1 / 2\left(\gamma^{2}\left\|w_{k}\right\|^{2}-\left\|z_{k}\right\|^{2}\right)$ and $V=\hat{\alpha} H$ is

$$
\alpha\left(H\left(x_{k+1}\right)-H\left(x_{k}\right)\right)=\frac{\beta}{2}\left(\gamma^{2}\left\|w_{k}\right\|^{2}-\left\|z_{k}\right\|^{2}\right)-\varphi_{k}
$$

where $\alpha=\hat{\alpha} \beta$ and $\varphi_{k}=\hat{\varphi}_{k} \beta$. Here $\alpha, \hat{\alpha}$ and $\beta$ are positive real numbers, $\varphi_{k}$ and $\hat{\varphi}_{k}$ are functions as in Definition 4. To improve performance of the closed loop system, we replace the equality sign in (23) with an inequality sign, i.e.

$$
\alpha\left(H\left(x_{k+1}\right)-H\left(x_{k}\right)\right) \leq \frac{\beta}{2}\left(\gamma^{2}\left\|w_{k}\right\|^{2}-\left\|z_{k}\right\|^{2}\right)-\varphi\left(x_{k}, w_{k}\right), \quad x_{k}=\left(q_{k}, p_{k}\right)
$$

then using the system equations (10) and, the first of the discrete-gradient conditions given in Definition 6 yields,

$$
\begin{aligned}
\alpha\left(H\left(x_{k+1}\right)-H\left(x_{k}\right)\right) & =\alpha\left[\begin{array}{c}
\bar{\nabla}_{q} H \\
\bar{\nabla}_{p} H
\end{array}\right]^{T}\left[\begin{array}{c}
q_{k+1}-q_{k} \\
p_{k+1}-p_{k}
\end{array}\right] \\
& =-\alpha T \bar{\nabla}_{p}^{T} H R_{1}\left(q_{k}, p_{k}\right) \bar{\nabla}_{p} H+\alpha T \bar{\nabla}_{p}^{T} H g_{1}\left(q_{k}\right) w_{k} \\
& \leq \frac{\beta}{2}\left(\gamma^{2} w_{k}^{T} w_{k}-y_{k}^{T} y_{k}\right)-\phi\left(\left(q_{k}, p_{k}\right), w_{k}\right) .
\end{aligned}
$$

The selection of the dissipation rate function as

$$
\varphi=\frac{1}{2}\left\|\gamma^{-1} \beta^{-1 / 2} \alpha T \bar{\nabla}_{p}^{T} H G_{1}-\gamma \beta^{1 / 2} w_{k}\right\|^{2}
$$

yields 


$$
\begin{aligned}
& -2 \alpha T \bar{\nabla}_{p}^{T} H R_{1}\left(q_{k}, p_{k}\right) \bar{\nabla}_{p} H+ \\
& +\beta\left(1+\gamma^{-2} \beta^{-2} \alpha^{2} T^{2}\right) \bar{\nabla}_{p}^{T} H g_{1}\left(q_{k}\right) g_{1}^{T}\left(q_{k}\right) \bar{\nabla}_{p} H \leq 0 .
\end{aligned}
$$

Finally selecting $\beta=\alpha T$ yields

$$
\begin{aligned}
& -2 \bar{\nabla}_{p}^{T} H R_{1}\left(q_{k}, p_{k}\right) \bar{\nabla}_{p} H+ \\
& +\left(1+\gamma^{-2}\right) \bar{\nabla}_{p}^{T} H g_{1}\left(q_{k}\right) g_{1}^{T}\left(q_{k}\right) \bar{\nabla}_{p} H \leq 0 .
\end{aligned}
$$

By Remark 1 and the Bounded Real Lemma, if this inequality holds than the $L_{2}$ gain of the system, from the disturbance input $w_{k}$ to the penalty output $z_{k}$ is less than or equal to $\gamma$. Now, it is easy to see that the inequality (26) holds if $R_{1}\left(q_{k}, p_{k}\right)$ satisfies (22).

If condition (22) is not satisfied then a feedback control has to be implemented. The use of PBC design allows to obtain any desired $R_{d}\left(q_{k}\right)$ in the form of

$$
R_{d}=\left[\begin{array}{lc}
0 & 0 \\
0 & g_{2}\left(q_{k}\right) K_{v} g_{2}^{T}\left(q_{k}\right)
\end{array}\right]
$$

selecting

$$
u_{k}=-K_{v} g_{2}^{T}\left(q_{k}\right) \bar{\nabla}_{p} H .
$$

This control law is called damping injection control law [2, 3, 4]. Selecting

$$
K_{v}=\frac{\left(1+\gamma^{-2}\right)}{2} g_{2}^{-1}\left(q_{k}\right) g_{1}\left(q_{k}\right) g_{1}^{T}\left(q_{k}\right) g_{2}^{-T}\left(q_{k}\right)
$$

yields $R_{d}=R$ with $R_{1}$ satisfying (22). Note that this selection is only possible for fully-actuated systems since it includes the inverse of the input port matrix.

\section{Remark 2}

When the open loop system has dissipation structure $R_{s y s}[2,3,4]$, the condition on the minimum required dissipation structure to be added to the system is obtained as,

$$
\underline{\sigma} R_{1 r}\left(q_{k}, p_{k}\right) \geq \bar{\sigma}\left(\frac{\left(1+\gamma^{-2}\right)}{2} g_{1}\left(q_{k}\right) g_{1}^{T}\left(q_{k}\right)-R_{1 s y s}\right)
$$

and one could select for fully-actuated systems

$$
K_{v}=g_{2}^{-1}\left(q_{k}\right)\left(\frac{\left(1+\gamma^{-2}\right)}{2} g_{1}\left(q_{k}\right) g_{1}^{T}\left(q_{k}\right)-R_{l s y s}\right) g_{2}^{-T}\left(q_{k}\right)
$$

to assign the dissipation structure that satisfies (22) to the closed-loop system.

The new result on the solution of the disturbance attenuation problem defined in Subsection 3.1 is presented in the following theorem. It is explained in Remark 3 that the control law constructed in this theorem adds a dissipation structure that satisfies (22) to the system. 


\section{Theorem 1}

Consider the discrete-time port-controlled Hamiltonian system given in (8)-(9), and suppose the system satisfies the following conditions.

I. The system is zero-state detectable.

II. The weighting matrix $d\left(q_{k}, p_{k}\right)$ has full rank.

III. The equilibrium point $x^{*}=0$ is a strict local minimizer of $H(x)$.

IV. There exist a discrete gradient, i.e., namely, $\bar{\nabla} H\left(x_{k+1}, x_{k}\right), x_{k}=\left\{q_{k}, p_{k}\right\} \in R^{2 n}$ which satisfies the conditions in Definition 6.

Then the feedback control law

$$
u_{k}=-\alpha T\left\{d_{k}^{T} d_{k}\right\}^{-1} g_{2}^{T}\left(q_{k}\right) \bar{\nabla}_{p} H
$$

where $d_{k}$ is such that

$$
d_{k}^{T} d_{k}=\left\{\left(\frac{\left(1+\gamma^{-2} \alpha^{2} T^{2}\right)}{\alpha T}\right) \tilde{d}^{T} \hat{g}_{2}^{-1}\left(q_{k}\right) \hat{g}_{1}\left(q_{k}\right) \hat{g}_{1}^{T}\left(q_{k}\right) \hat{g}_{2}^{-T}\left(q_{k}\right) \tilde{d}\right\}^{-1}
$$

$\tilde{d} \in \mathfrak{R}^{m_{1} \times n}$ is a full rank constant matrix, $\hat{g}_{1}\left(q_{k}\right) \in \mathfrak{R}^{n \times m_{1}}$ and $\hat{g}_{2}\left(q_{k}\right) \in \mathfrak{R}^{n \times m_{2}}$ are full rank matrices such that $\sigma_{i}\left(\hat{g}_{1}\left(q_{k}\right)\right) \geq \sigma_{i}\left(g_{1}\left(q_{k}\right)\right), \sigma_{i}\left(\hat{g}_{2}\left(q_{k}\right)\right) \leq \sigma_{i}\left(g_{2}\left(q_{k}\right)\right), i=1, \ldots, n$ and $\alpha T \underline{\sigma}\left(\tilde{d}^{T} \tilde{d}\right) \geq 1$ renders the $L_{2}$ gain of the closed loop system, from the disturbance input $w_{k}$ to penalty output $z_{k}$, equal to or less than $\gamma$.

\section{Proof}

If $d_{k}$ is taken as in (33) the inequality given in (20) becomes

$$
\begin{aligned}
& \left(1+\alpha^{2} \gamma^{-2} T^{2}\right) g_{l}\left(q_{k}\right) g_{l}^{T}\left(q_{k}\right)-\alpha T\left(1+\alpha^{2} \gamma^{-2} T^{2}\right) g_{2}\left(q_{k}\right) \tilde{d}^{T} \hat{g}_{2}^{-1}\left(q_{k}\right) \hat{g}_{l}\left(q_{k}\right) \hat{g}_{l}^{T}\left(q_{k}\right) \hat{g}_{2}^{-T}\left(q_{k}\right) \tilde{d} g_{2}^{T}\left(q_{k}\right) \\
& -2 \alpha T R_{l}\left(q_{k}, p_{k}\right) \leq 0 .
\end{aligned}
$$

Since $\sigma_{i}\left(\hat{g}_{1}\left(q_{k}\right)\right) \geq \sigma_{i}\left(g_{1}\left(q_{k}\right)\right), \sigma_{i}\left(\hat{g}_{2}\left(q_{k}\right)\right) \leq \sigma_{i}\left(g_{2}\left(q_{k}\right)\right), i=1, \ldots, n$, this inequality is satisfied for any $\alpha \underline{\sigma}\left(\tilde{d}^{T} \tilde{d}\right) \geq 1 / T$ which proves the claim.

\section{Remark 3}

Selecting $\alpha=1 / T$ in the control law (32)-(33) yields the damping injection control law $u_{k}=-K_{v} g_{2}{ }^{T}\left(q_{k}\right) \bar{\nabla}_{p} H$ with $K_{v}=\left(1+\gamma^{-2}\right) \tilde{d}^{T} \hat{g}_{2}^{-1}\left(q_{k}\right) \hat{g}_{1}\left(q_{k}\right) \hat{g}_{1}^{T}\left(q_{k}\right) \hat{g}_{2}^{-T}\left(q_{k}\right) \tilde{d}$ which adds to the system the dissipation structure $R_{1}\left(q_{k}, p_{k}\right)=\left(1+\gamma^{-2}\right) g_{2}\left(q_{k}\right) \tilde{d}^{T} \hat{g}_{2}^{-1}\left(q_{k}\right) \hat{g}_{1}\left(q_{k}\right) \hat{g}_{1}^{T}\left(q_{k}\right) \hat{g}_{2}^{-T}\left(q_{k}\right) \tilde{d} g_{2}^{T}\left(q_{k}\right)$. Considering the conditions given in Theorem 1 on $\hat{g}_{1}\left(q_{k}\right), \hat{g}_{2}\left(q_{k}\right)$ and $\tilde{d}$ it can be seen that this dissipation structure satisfies the condition (22). Note that the control law (32)-(33) assigns to the system a dissipation structure that satisfies (22) in the case of fully actuated systems. If $g_{1}^{-1}\left(q_{k}\right)$ and $g_{2}^{-1}\left(q_{k}\right)$ exist, $\hat{g}_{1}\left(q_{k}\right), \hat{g}_{2}\left(q_{k}\right), \tilde{d}$ can be selected as $\hat{g}_{1}\left(q_{k}\right)=g_{1}\left(q_{k}\right), \hat{g}_{2}\left(q_{k}\right)=g_{2}\left(q_{k}\right), \tilde{d}=I_{n}$ and selecting $\alpha=1 / T$ in the control law (32)-(33) yields a damping injection control law with 
$K_{v}=\left(1+\gamma^{-2}\right) g_{2}^{-1}\left(q_{k}\right) g_{1}\left(q_{k}\right) g_{1}^{T}\left(q_{k}\right) g_{2}^{-T}\left(q_{k}\right) \quad$ that assigns the dissipation structure $R_{1}\left(q_{k}, p_{k}\right)=\left(1+\gamma^{-2}\right) g_{1}\left(q_{k}\right) g_{1}^{T}\left(q_{k}\right)$ which satisfies the condition (22). $\square$

\section{Remark 4}

To obtain a less conservative controller for the systems which have already some dissipation structure $R_{1}, d_{k}$ can be selected such that

$$
d_{k}^{T} d_{k}=\left(\frac{1}{\alpha T} \tilde{d}^{T} \hat{g}_{2}^{-1}\left(q_{k}\right)\left(1+\gamma^{-2} \alpha^{2} T^{2}\right) \hat{g}_{1}\left(q_{k}\right) \hat{g}_{1}^{T}\left(q_{k}\right)-2 R_{1} \hat{g}_{2}^{-T}\left(q_{k}\right) \tilde{d}\right)^{-1}
$$

where $\tilde{d}_{k}^{T} \tilde{d}_{k}=I_{m}$ in the control law (32). To be able to see that this selection gives a less conservative controller, consider the case $\hat{g}_{1}^{-1}=g_{1}^{-1} \in \mathfrak{R}^{n \times n}$ and $\hat{g}_{2}^{-1}=g_{2}^{-1} \in \mathfrak{R}^{n \times n}$ (i.e. fully-actuated systems) and select $\tilde{d}=I_{n}$. Thus, the inequality (20) becomes

$$
\begin{aligned}
& \left(1+\alpha^{2} \gamma^{-2} T^{2}\right) g_{l}\left(q_{k}\right) g_{l}^{T}\left(q_{k}\right)-\alpha T\left(1+\alpha^{2} \gamma^{-2} T^{2}\right) g_{l}\left(q_{k}\right) g_{l}^{T}\left(q_{k}\right) \\
& +2 \alpha T R_{l}\left(q_{k}, p_{k}\right)-2 \alpha T R_{l}\left(q_{k}, p_{k}\right) \leq 0
\end{aligned}
$$

and this inequality is satisfied for $\alpha=1 / T$ with the equality sign.

The new results on the robust disturbance attenuation are now stated. The corollary gives a lower bound on the dissipation that the uncertain system needs to have so that its $L_{2}$ gain from the disturbance input to the output is at most $\gamma$.

\section{Corollary}

Consider the uncertain discrete-time port-controlled Hamiltonian system (17)-(18) with $u_{k}=0$, $k=0,1,2, \ldots$. If

$$
\underline{\sigma}\left(R_{1}\left(q_{k}, p_{k}\right)\right) \geq \bar{\sigma}\left(\frac{\left(1+\gamma^{-2}\right)}{2}\left(\begin{array}{c}
g_{w}\left(q_{k}\right) g_{w}^{T}\left(q_{k}\right)+ \\
+g_{u}\left(q_{k}\right) g_{u}^{T}\left(q_{k}\right)
\end{array}\right)\right), \forall q_{k}, p_{k}
$$

then the $L_{2}$ gain of the system, from the disturbance input $w_{k}$ to the penalty output $z_{k}$ defined in (11) is less than or equal to $\gamma$.

Proof: Applying Proposition 1 to the system (17-18) yields (36).

Note that, a dissipation structure that satisfies this condition can be assigned for fully-actuated system, namely if $g_{2}^{-1}\left(q_{k}\right)$ exists, using the damping injection control law (28) by selecting

$$
K_{v}=\frac{\left(1+\gamma^{-2}\right)}{2} I+g_{u}^{-1}\left(q_{k}\right) g_{w}\left(q_{k}\right) g_{w}^{T}\left(q_{k}\right) g_{u}^{-T}\left(q_{k}\right)
$$

\section{Remark 5}

When the open loop system has dissipation structure $R_{s y s}[2,3,4]$, the condition on the minimum required dissipation to be added is obtained as 


$$
\underline{\sigma} R_{1 r}\left(q_{k}, p_{k}\right) \geq \bar{\sigma}\left(\frac{\left(1+\gamma^{-2}\right)}{2}\left(\begin{array}{c}
g_{w}\left(q_{k}\right) g_{w}^{T}\left(q_{k}\right)+ \\
+g_{u}\left(q_{k}\right) g_{u}^{T}\left(q_{k}\right)
\end{array}\right)-R_{1 s y s}\right) \text {. }
$$

For the case of fully-actuated systems one can select

$$
K_{v}=g_{2}^{-1}\left(q_{k}\right)\left(\frac{\left(1+\gamma^{-2}\right)}{2}\left(\begin{array}{c}
g_{w}\left(q_{k}\right) g_{w}^{T}\left(q_{k}\right)+ \\
+g_{u}\left(q_{k}\right) g_{u}^{T}\left(q_{k}\right)
\end{array}\right)-R_{l s y s}\right) g_{2}^{-T}\left(q_{k}\right),
$$

which assigns a dissipation structure which satisfies (36).

The following theorem presents the solution of the robust disturbance attenuation problem considered in Section 3.2, namely provides a controller that assigns the closed loop system a dissipation structure that satisfies (36). This theorem can be used also for under-actuated systems.

\section{Theorem 2}

Consider the discrete-time port-controlled Hamiltonian system given in (17)-(18), and suppose the system satisfies the following conditions.

I. The system is zero-state detectable.

II. The weighting matrix $d\left(q_{k}, p_{k}\right)$ has full column rank.

III. The equilibrium point $x^{*}=0$ is a strict local minimizer of $H(x)$.

IV. There exist a discrete gradient, i.e., namely, $\bar{\nabla} H\left(x_{k+1}, x_{k}\right), x_{k}=\left\{q_{k}, p_{k}\right\} \in R^{2 n}$ which satisfies the conditions in Definition 6.

Then a robust optimal disturbance attenuation control law can be given by

$$
u_{r k}=-\alpha T\left\{d_{r k}^{T} d_{r k}\right\}^{-1} g_{2}^{T}\left(q_{k}\right) \bar{\nabla}_{p} H
$$

where the parameter $d_{r k}$ is such that

$$
d_{r k}^{T} d_{r k}=\left(\frac{\left(1+\gamma^{-2} \alpha^{2} T^{2}\right)}{\alpha T} \tilde{d}_{r}^{T}\left(I+\hat{g}_{u}^{-1}\left(q_{k}\right) \hat{g}_{w}\left(q_{k}\right) \hat{g}_{w}^{T}\left(q_{k}\right) \hat{g}_{u}^{-T}\left(q_{k}\right)\right) \tilde{d}_{r}\right)^{-1}
$$

$\tilde{d}_{r} \in \mathfrak{R}^{m_{1} \times n}$ is a full rank constant matrix, $\hat{g}_{1}\left(q_{k}\right) \in \mathfrak{R}^{n \times m_{1}}$ and $\hat{g}_{2}\left(q_{k}\right) \in \mathfrak{R}^{n \times m_{2}}$ are full-rank matrices such that $\sigma_{i}\left(\hat{g}_{1}\left(q_{k}\right)\right) \geq \sigma_{i}\left(g_{1}\left(q_{k}\right)\right), \quad \sigma_{i}\left(\hat{g}_{2}\left(q_{k}\right)\right) \leq \sigma_{i}\left(g_{2}\left(q_{k}\right)\right), i=1, \ldots, n$ and $\alpha T \underline{\sigma}\left(\tilde{d}_{r}^{T} \tilde{d}_{r}\right) \geq 1$, renders the $L_{2}$ gain of the closed loop system, from the disturbance input $w_{k}$ to penalty output $z_{k}$, equal to or less than $\gamma \square$.

\section{Proof}

The proof is similar to the proof of Theorem 1. If $d_{k}$ is taken as in (39) the inequality (21) becomes 


$$
\begin{aligned}
& \left(1+\alpha^{2} \gamma^{-2} T^{2}\right) g_{u}\left(q_{k}\right) g_{u}^{T}\left(q_{k}\right)+\left(1+\alpha^{2} \gamma^{-2} T^{2}\right) g_{w}\left(q_{k}\right) g_{w}^{T}\left(q_{k}\right)-\alpha T\left(1+\gamma^{-2} \alpha^{2} T^{2}\right) g_{u}\left(q_{k}\right) \tilde{d}_{r}^{T} \tilde{d}_{r} g_{u}^{T}\left(q_{k}\right) \\
& \left.-\alpha T\left(1+\gamma^{-2} \alpha^{2} T^{2}\right) g_{u}\left(q_{k}\right) \tilde{d}_{r}^{T} \hat{g}_{u}^{-1}\left(q_{k}\right) \hat{g}_{w}\left(q_{k}\right) \hat{g}_{w}^{T}\left(q_{k}\right) \hat{g}_{u}^{-T}\left(q_{k}\right) \tilde{d}_{r} g_{u}^{T}\left(q_{k}\right)\right)-2 \alpha T R_{1}\left(q_{k}, p_{k}\right) \leq 0
\end{aligned}
$$

Since $\sigma_{i}\left(\hat{g}_{1}\left(q_{k}\right)\right) \geq \sigma_{i}\left(g_{1}\left(q_{k}\right)\right), \sigma_{i}\left(\hat{g}_{2}\left(q_{k}\right)\right) \leq \sigma_{i}\left(g_{2}\left(q_{k}\right)\right), i=1, \ldots, n$, this inequality is satisfied for any $\alpha \underline{\sigma}\left(\tilde{d}_{r}^{T} \tilde{d}_{r}\right) \geq 1 / T$ which proves the claim.

\section{Remark 6}

Selecting $\alpha=1 / T$ in the control law (38)-(39) yields the damping injection control law $u_{k}=-K_{v} g_{2}{ }^{T}\left(q_{k}\right) \bar{\nabla}_{p} H \quad$ with $\quad K_{v}=\left(1+\gamma^{-2}\right) \tilde{d}_{r}^{T}\left(I+\hat{g}_{u}^{-1}\left(q_{k}\right) \hat{g}_{w}\left(q_{k}\right) \hat{g}_{w}^{T}\left(q_{k}\right) \hat{g}_{u}^{-T}\left(q_{k}\right)\right) \tilde{d}_{r}$ which adds the dissipation structure $R_{1}\left(q_{k}, p_{k}\right)=\left(1+\gamma^{-2}\right) g_{u}\left(q_{k}\right) \tilde{d}_{r}^{T} \tilde{d}_{r} g_{u}^{T}\left(q_{k}\right)+g_{u}\left(q_{k}\right) \tilde{d}_{r}^{T} \hat{g}_{u}^{-1}\left(q_{k}\right) \hat{g}_{w}\left(q_{k}\right) \hat{g}_{w}^{T}\left(q_{k}\right)$ $\hat{g}_{u}^{-T}\left(q_{k}\right) \tilde{d}_{r} g_{u}^{T}\left(q_{k}\right)$ to the system. Considering the conditions given in Theorem 2 on $\hat{g}_{1}\left(q_{k}\right), \hat{g}_{1}\left(q_{k}\right)$

and $\tilde{d}$, it can be seen that this dissipation structure satisfies the condition (36). Note that, it is easier to see that the control law (38)-(39) assigns to the system a dissipation structure which satisfies (36) in the case of fully actuated systems. If $g_{1}^{-1}\left(q_{k}\right)$ and $g_{2}^{-1}\left(q_{k}\right)$ exist, $\hat{g}_{1}\left(q_{k}\right), \hat{g}_{2}\left(q_{k}\right), \tilde{d}$ can be selected as $\hat{g}_{1}\left(q_{k}\right)=g_{1}\left(q_{k}\right), \hat{g}_{2}\left(q_{k}\right)=g_{2}\left(q_{k}\right), \tilde{d}=I_{n}$ and selecting $\alpha=1 / T$ in the control law (38)(39) yields a damping injection control law with $K_{v}=\left(1+\gamma^{-2}\right) I+g_{u}^{-1}\left(q_{k}\right) g_{w}\left(q_{k}\right) g_{w}^{T}\left(q_{k}\right) g_{u}^{-T}\left(q_{k}\right)$ that assign the dissipation structure $R_{1}\left(q_{k}, p_{k}\right)=\left(1+\gamma^{-2}\right) g_{u}\left(q_{k}\right) g_{u}^{T}\left(q_{k}\right)+g_{w}\left(q_{k}\right) g_{w}^{T}\left(q_{k}\right) \quad$ which satisfies the condition (36)..

\section{Remark 7}

Similarly to Remark 4, for the systems which have already some dissipation $R_{1}, d_{k}$ can be selected such that

$$
d_{r k}^{T} d_{r k}=\left(\frac{1}{\alpha T} \tilde{d}_{r}^{T} \hat{g}_{u}^{-1}\left(q_{k}\right)\left(\left(1+\gamma^{-2} \alpha^{2} T^{2}\right)\left(\hat{g}_{u}\left(q_{k}\right) \hat{g}_{u}^{T}+\hat{g}_{w}\left(q_{k}\right) \hat{g}_{w}^{T}\left(q_{k}\right)\right)-2 R_{1}\right) \hat{g}_{u}^{-T}\left(q_{k}\right) \tilde{d}_{r}\right)^{-1}
$$

with $\tilde{d}_{k}^{T} \tilde{d}_{k}=I_{m}$ to obtain a less conservative controller.

\section{EXAMPLE and SIMULATIONS}

To analyse the performance of the proposed controller we considered the ball and beam system illustrated in Figure 1, where $x$ is the ball position, $\theta$ the angle of the bar and $L$ is the bar length. The dynamics of the ball and beam system can be described by the Euler-Lagrange equations [24]

$$
\begin{aligned}
& \ddot{q}_{1}+g \sin q_{2}-q_{1} \dot{q}_{2}^{2}=0, \\
& \left(L+q_{1}^{2}\right) \ddot{q}_{2}+2 q_{1} \dot{q}_{1} \dot{q}_{2}+g q_{1} \cos \left(q_{2}\right)=u .
\end{aligned}
$$

These equations are scaled in time and masses. $L^{2}$ explicitly appears in the equations since it is a factor of the moment of the inertia of the bar, for details see [24]. These dynamics without dissipation can be expressed in the Hamiltonian system formalism as follows 


$$
\left[\begin{array}{c}
\dot{q} \\
\dot{p}
\end{array}\right]=(J-R(q, p)) \nabla H+\left[\begin{array}{l}
0 \\
G
\end{array}\right] u, R(q, p)=0
$$

with $H(q, p)=\frac{1}{2} p^{T} M(q)^{-1} p+P(q)$ in which

$$
M=\left[\begin{array}{cc}
1 & 0 \\
0 & L^{2}+q_{1}^{2}
\end{array}\right], \quad P q=g q_{1} \sin q_{2}, G^{T}=0 \quad 1 .
$$

Note that the state variables are the position of the ball $q_{1}=x$, the vertical angle of the bar $q_{2}=\theta$, $p_{1}=\dot{q}_{1}$, and $p_{2}=\left(L+q_{1}^{2}\right) \dot{q}_{2}$.

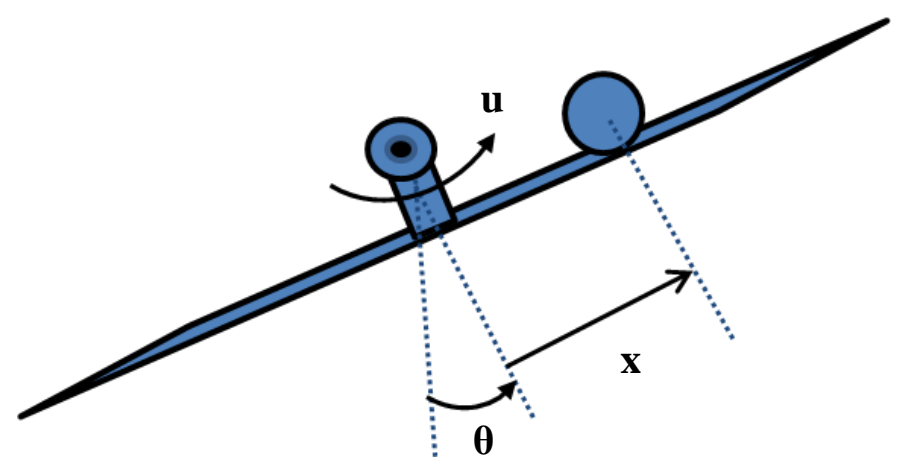

Figure 1. The ball and beam system.

We run simulations for the system obtained applying the energy shaping control law designed in [24] that assigns a desired energy function to the system (41)-(42) so that $q_{1^{*}}=q_{2^{*}}=0$ is the only equilibrium. We consider this closed loop system to test the performance of the disturbance attenuation control laws (32)-(33) and (38)-(39), because the objective of the control is to attenuate the disturbance. The matrices $M_{d}, J_{d}$ and the shaped potential energy function $V_{d}$ completely describe the considered closed loop (desired) dynamics, as follows,

$$
\begin{aligned}
& M_{d}=L^{2}+q_{1}^{2}\left[\begin{array}{cc}
\sqrt{\frac{2}{L^{2}+q_{1}^{2}}} & 1 \\
1 & \sqrt{2 L^{2}+q_{1}^{2}}
\end{array}\right], J_{2} q, p=\left[\begin{array}{ccc}
0 & j & p, q \\
-j & p, q & 0
\end{array}\right] \\
& V_{d} q=g 1-\cos q_{2}+\Phi z q
\end{aligned}
$$

where $q=q_{1} \quad q_{2}{ }^{T}, p=p_{1} \quad p_{2}{ }^{T}, j=q_{1}\left(p_{1}-\sqrt{\frac{2}{L^{2}+q_{1}^{2}}} p_{2}\right), z q=q_{2}-\arcsin h\left(\frac{q_{1}}{L}\right) / \sqrt{2}$ and $\Phi z=\frac{K_{p}}{2} z^{2}$. We also consider the case in which an external disturbance acts on the system and there is uncertainty in the mass of the ball and the bar. Hence the Hamiltonian model of the system with external disturbance and uncertainty considered in the simulations is

$$
\left[\begin{array}{c}
\dot{q} \\
\dot{p}
\end{array}\right]=\left(J-R_{d}(q, p)\right) \nabla \tilde{H}_{d}(q, p)+\left[\begin{array}{c}
0 \\
g_{w}(q)
\end{array}\right] w+\left[\begin{array}{c}
0 \\
g_{u}(q)
\end{array}\right] u,
$$


where $\quad g_{w}^{T}(q)=g_{u}^{T}(q)=0 \quad 1, \quad R_{d}=0, \quad \tilde{H}_{d}(q, p)=\frac{1}{2} p^{T} \tilde{M}_{d}(q)^{-1} p+\tilde{P}_{d}(q), \quad \tilde{M}_{d}(q)=M_{d}(q)+$ $\Delta M_{d}(q)$ and $\tilde{P}_{d}(q)=P_{d}(q)+\Delta P_{d}(q)$, yielding

$$
\Delta H_{d}(q, p)=\frac{1}{2} p^{T} \Delta M_{d}(q)^{-1} p+\Delta P_{d}(q)
$$

The system parameters are taken as $K_{p}=1.0, L=10$ and $g=0.98 \mathrm{~ms}^{-2}$ as in [24]. Simulations are run for $T=0.1$. The disturbance input considered is $w=1 N$ for $t \in[5,7]$ and zero otherwise. The time response of the nominal system under the disturbance is shown in Figure 1. Figure 2 illustrates the closed loop performances for three cases: attenuation without presence of uncertainty, attenuation in presence of uncertainty, and robust attenuation in presence of uncertainty. $60 \%$ per cent of uncertainty is considered in the masses of ball and bar (60\% less than their nominal values). The robust control law to achieve $\gamma=0.04$ is obtained as follows: $\hat{g}_{u}$ and $\hat{g}_{w}$ are taken as $\hat{g}_{u}=I_{2}$, $\hat{g}_{w}=I_{2}$. Thus, selecting $\tilde{d}^{T}=0 \quad 1.01$ and $\alpha=1 / T$, yields

$$
\begin{aligned}
& d_{k}^{T} d_{k}{ }^{-1}=\frac{\left(1+\gamma^{-2} \alpha^{2} T^{2}\right)}{\alpha T} \tilde{d}^{T}\left(I_{2}+\hat{g}_{u}^{-1}\left(q_{k}\right) \hat{g}_{w}\left(q_{k}\right) \hat{g}_{w}^{T}\left(q_{k}\right) \hat{g}_{u}^{-T}\left(q_{k}\right)\right) \tilde{d} \\
& =\left(1+\gamma^{-2}\right) \tilde{d}^{T}\left(I_{2}+I_{2}\right) \tilde{d}=2\left(1+\gamma^{-2}\right) \tilde{d}^{T} \tilde{d} \\
& =2\left(1+(0.04)^{-2}\right) 0 \quad 1.01\left[\begin{array}{c}
0 \\
1.01
\end{array}\right]=1277.2
\end{aligned}
$$

Substituting this value and $\alpha T=1$ in the control law (38) yields

$$
\begin{aligned}
u_{k} & =-\alpha T\left\{d_{k}^{T} d_{k}\right\}^{-1} g_{2}{ }^{T}\left(q_{k}\right) \bar{\nabla}_{p} H \\
& =-1277.20 \quad 1 \bar{\nabla}_{p} H=-1277.2 \bar{\nabla}_{p_{2}} H .
\end{aligned}
$$

Note that $\tilde{d}^{T} \tilde{d}=1.0201$, hence $\underline{\sigma}\left(\tilde{d}^{T} \tilde{d}\right)=1.0201 \geq 1$ and $\alpha T \underline{\sigma}\left(\tilde{d}^{T} \tilde{d}\right)=1.0201 \geq 1$.

When the uncertainty is ignored, $d_{k}$ is obtained selecting $\hat{g}_{u}=I_{2}, \hat{g}_{w}=I_{2}, \tilde{d}^{T}=0 \quad 1.01$ and $\alpha=1 / T$, and substituting (33) as follows

$$
\begin{aligned}
& d_{k}^{T} d_{k}{ }^{-1}=\left(\frac{\left(1+\gamma^{-2} \alpha^{2} T^{2}\right)}{\alpha T}\right) \tilde{d}^{T} \hat{g}_{2}^{-1}\left(q_{k}\right) \hat{g}_{1}\left(q_{k}\right) \hat{g}_{1}^{T}\left(q_{k}\right) \hat{g}_{2}^{-T}\left(q_{k}\right) \tilde{d} \\
& =\left(1+\gamma^{-2}\right) \tilde{d}^{T} \tilde{d} \\
& =\left(1+(0.04)^{-2}\right) 0 \quad 1.01\left[\begin{array}{c}
0 \\
1.01
\end{array}\right]=637.6
\end{aligned}
$$

Substituting this value and $\alpha T=1$ in the control law (32) yields

$$
\begin{aligned}
& u_{r k}=-\alpha T\left\{d_{r k}^{T} d_{r k}\right\}^{-1} g_{2}{ }^{T}\left(q_{k}\right) \bar{\nabla}_{p} H \\
& =-637.60 \quad 1 \bar{\nabla}_{p} H=-637.6 \bar{\nabla}_{p_{2}} H
\end{aligned}
$$

since $\alpha T=1$. 
Simulations have been carried out for the control laws (43) and (44). The results are given as a comparison between robust design and the design performed ignoring uncertainty for the cases of the system with uncertainty and without uncertainty. The generalized positions and the momentums of the controlled system versus time are illustrated in the Figure 2 and Figure 3. As can be seen from these figures, the controller which is designed ignoring uncertainties is not able to attenuate the disturbance while the controller obtained with the robust design successfully attenuates the disturbance as expected from the theoretical results. The simulations are run with $T=0.01$.

\section{CONCLUSIONS}

The discrete-time disturbance attenuation and robust disturbance attenuation problems for port controlled Hamiltonian systems are considered. The condition for the lower bound on the amount of dissipation that a system needs to possess to have a disturbance attenuation level equal to or less than $\gamma$ has been given and the discrete-time damping injection control law that assigns a dissipation structure that satisfies this condition has been given for fully actuated systems. New solutions for the disturbance attenuation and robust disturbance attenuation problems are presented for the worst case that the system has no dissipation. These solutions provide controllers removing the need to solve HJI for both fully-actuated and under-actuated systems. Note that, less conservative controllers are also given for systems possessing some dissipation. The relation between these control laws and damping injection control laws is also established and damping injection equivalents of the controllers are provided. The proposed controllers are applied to the ball and beam system. Simulation results illustrate that in the absence of uncertainty the proposed disturbance attenuation controller successfully attenuates the disturbance whereas in the presence of uncertainty the proposed robust disturbance attenuation control law need to be used.
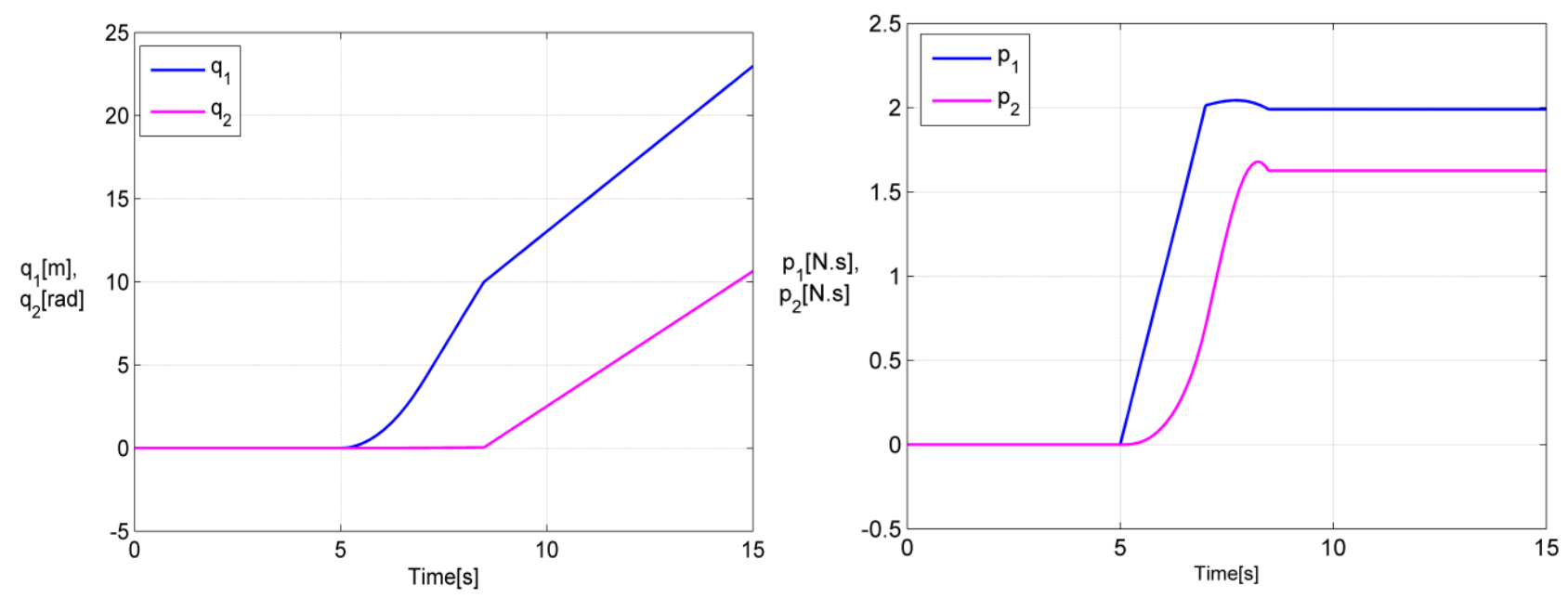

Fig. 2. Time histories of $q$ and $p$ for the uncontrolled ball and beam system in the presence of disturbance. 

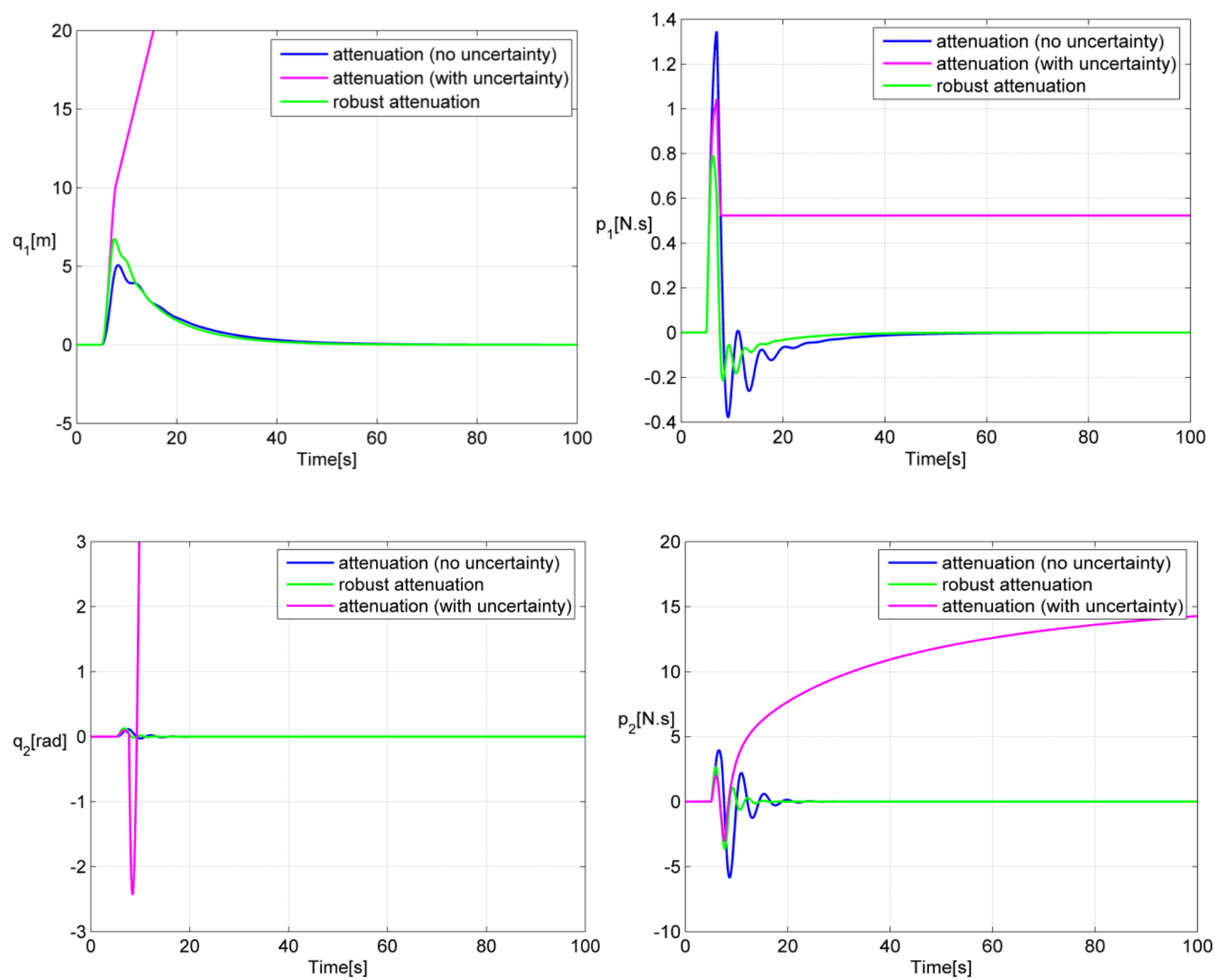

Fig. 3. Time histories of $q$ and $p$ for the closed-loop system in the presence of disturbance.

\section{REFERENCES}

[1] Navarro-López EM. Dissipativity and Passivity-Related Properties in Nonlinear Discrete-Time Systems. Ph.D. Thesis: Institute of Industrial and Control Engineering, 2002.

[2] Van der Schaft A. $L_{2}$-Gain and Passivity Techniques in Nonlinear Control. Springer: London, 2000.

[3] Ortega R, Van der Schaft AJ, Maschke B, Escobar G. Interconnection and damping assignment passivitybased control of port-controlled Hamiltonian systems. Automatica 2002; 38: 585-596.

[4] Ortega R, Garcia-Canseco E. Interconnection and Damping Assignment Passivity-Based Control. European Journal of Control 2004; 10 (5): 432-450.

[5] Isidori A, Astolfi A. "Disturbance Attenuation and $\mathrm{H}_{\infty}$-Control via Measurement Feedback in Nonlinear Systems.” IEEE Trans. on Automatic Control 1992; 37 (9): 1283-1293.

[6] Isidori A, Kang W. $\mathrm{H}_{\infty}$-Control via Measurement Feedback for General Nonlinear Systems. IEEE Trans. on Automatic Control 1995; 40 (3): 466-472.

[7] Lu G, Zheng Y, Ho DWC. Nonlinear robust $\mathrm{H}_{\infty}$-Control via Dynamic Output Feedback. Systems \& Control Letters 2000; 39: 193-202.

[8] Xi Z, Cheng D. Passivity-Based Stabilization and $\mathrm{H}_{\infty}$ Control of the Hamiltonian Control Systems with Dissipation and Its Applications to Power Systems. International Journal of Control 2000; 73 (18): 16861691. 
[9] Mei S, Shen T, Hu W, Lu Q, Sun L. Robust $\mathrm{H}_{\infty}$-Control of a Hamiltonian system with uncertainty and its application to a multi-machine power system. IET Control Theory and Application 2005; 152 (2):202-210.

[10] Byrnes CI, Lin W. Losslessness, feedback equivalence, and the global stabilization of discrete-time nonlinear systems. IEEE Transaction on Automatic and Control 39 (1) 1994:83-98.

[11] Lin W, Byrnes CI. Discrete-time Nonlinear $\mathrm{H}_{\infty}$ Control with Measurement Feedback. Automatica 1995; 31 (3): 419-434.

[12] Sengör NS. Energy related concepts in nonlinear systems, Ph.D. Thesis: Istanbul Technical University, Institute of Science and Technology, 1995.

[13] Göknar IC, Sengör NS. Discrete-time version of Kalman-Yacubovitch-Popov Lemma for Nonlinear Systems. Proceedings of the ICECS, Lisbon, Portugal 1998:1054-1056.

[14] Nesic D, Teel AR. A framework for stabilization of nonlinear sampled-data systems based on their approximate discrete-time models. IEEE Transaction on Automatic Control 2004; 49 (7): 1103-1122.

[15] Laila DS, Astolfi A. Discrete-time IDA-PBC design for separable Hamiltonian systems. Proceedings of the 16th IFAC World Congress, Prague, 3-8 July 2005: 539-539.

[16] Laila DS, Astolfi A. Direct Discrete-time Design for Sampled-data Hamiltonian Control Systems. Proceedings of the 3rd IFAC Workshop on Lagrangian and Hamiltonian Methods for Nonlinear Control, Nogoya, Japan, July 2006: 57-62.

[17] Laila DS, Astolfi A. Discrete-time IDA-PBC design for separable Hamiltonian systems. Proceedings of the American Control Conference, Minneapolis, Minnesota USA, June 14-16 2006: 188-193.

[18] Gören-Sümer L, Yalçın Y. Gradient Based Discrete-Time Modeling and Control of Hamiltonian Systems. Proceedings of the 17th IFAC World Congress, Seoul, Korea, 6-11 July 2008; 212-217.

[19] Yalçın Y, Gören-Sümer L. Direct Discrete-Time Control of Port Controlled Hamiltonian Systems. Turkish Journal of Electrical Engineering and Computer Sciences 2010; 18 (5): 913-924.

[20] Yalçın Y, Gören-Sümer L. Disturbance Attenuation in Hamiltonian Systems via Direct Discrete Time Design. Proceedings of the IFAC World Congress, Seoul, Korea, 6-11 July 2008; 194-199.

[21] Yalçın Y, Gören-Sümer L. Robust Disturbance Attenuation in Hamiltonian Systems via Direct Digital Control. Proceedings of the European Control Conference, Budapest, Hungary, 23-26 August 2009; 2277-2282.

[22] Gonzalez O, Simo JC. On the stability of symplectic and energy-momentum algorithms for non-linear Hamiltonian systems with symmetry. Computer Methods in Applied Mechanics and Engineering 1996; 134:197-222.

[23] Hill DJ, Moylan PJ. Dissipative dynamical systems: basic input-output and state properties. Journal of The Franklin Institute 1980; 309 (5): 327-357.

[24] Hauser J, Sastry S, Kokotovic P. Nonlinear control via approximate input-output linearization: The ball and beam example. IEEE Transaction on Automatic Control 1992; 37: 392-398.

[25] Ortega R, Spong MW, Gomez-Estern F, Blankenstein G. Stabilization of a class of underactuated mechanical systems via interconnection and damping assignment. IEEE Transactions on Automatic Control 2002; 47:1218-1233. 